\title{
Análisis de la composición de especies en la pesquería de cerco orientada a la captura de anchoveta (Engraulis ringens) en la Zona Norte de Chile
}

\author{
Analysis of the species composition in the purse seine fishery targeting anchovy (Engraulis ringens) in \\ the Northern Chile
}

\section{Carola Hernández-Santoro ${ }^{1 *}$ y Benjamín Suárez-Ahumada ${ }^{1}$}

${ }^{1}$ Instituto de Fomento Pesquero (IFOP), Blanco 839, Valparaíso, Chile. *Autor corresponsal: carola.hernandez@ifop.cl

\begin{abstract}
The composition and species proportion of catch were analyzed in the industrial and artisanal purse seine fishery occurred in the north of Chile. Biological and fishing information obtained on board fishing vessels between 2004 and 2012 through the Monitoring Program of Pelagic Fisheries in North Zone of Chile were used in this study. 9639 fishing hauls were analyzed. Four catch profiles explained the $34.27 \%$ of variance. The catch profiles were mainly composed by anchovy Engraulis ringens, jack mackerel Trachurus murphyi and mackerel Scomber japonicus. Anchovy was the dominant species. Only one catch profile grouped non-target species. Using GLM, the influence of type of fleet, temporal variability (year, month and trimester of arrival), day period, geographic position, sea surface temperature, fishing zone and coast distance in the catch profiles composition were analyzed. The trimester of arrival, day period, fishing zone, coast distance and sea surface temperature were the variables with greater statistical influence on catch profiles. A low proportion of non-target species was observed, influenced mainly by the fishing zone and the period of the day.
\end{abstract}

Key words: Engraulis ringens, catch profiles, catch composition, northern Chile, GLM, fishery

Resumen.- Se analizó la composición y proporción de especies en las capturas de la pesquería industrial y artesanal que opera con red de cerco de la zona norte de Chile. En este estudio se utilizó información biológico-pesquera obtenida a bordo de embarcaciones pesqueras entre los años 2004 y 2012, a través del Programa de Seguimiento de Pesquería Pelágicas de la Zona Norte de Chile. Se analizaron 9.639 lances de pesca. Se determinaron cuatro perfiles se captura que explicaron un $34,27 \%$ de la varianza. Los perfiles estuvieron compuestos principalmente por anchoveta Engraulis ringens, jurel Trachurus murphyi y caballa Scomber japonicus, con un alto porcentaje de anchoveta. Sólo un perfil presentó especies que componen la fauna acompañante. Se examinó la influencia del tipo de flota, la variabilidad temporal (año, mes y trimestre de recalada), el periodo del día, la posición geográfica, la temperatura superficial del lance, la zona de pesca y la distancia a la costa en la composición de los perfiles de captura utilizando modelos lineales generalizados. Las variables con mayor influencia estadística sobre los perfiles de captura fueron el trimestre de recalada, el periodo del día, la zona, la distancia a la costa y la temperatura. Se observó una baja proporción de especies no objetivo, influenciadas principalmente por la zona de pesca y el período del día.

Palabras clave: Engraulis ringens, perfiles de captura, composición especies, norte-Chile, GLM

\section{INTRODUCCIÓN}

En la zona norte de Chile, entre Arica y Antofagasta $\left(18^{\circ} 23^{\prime} \mathrm{S}\right.$ y $\left.24^{\circ} 00^{\prime} \mathrm{S}\right)$, la pesquería de cerco se ha orientado principalmente a la extracción de pequeños pelágicos, tales como anchoveta (Engraulis ringens) y sardina española (Sardinops sagax), además de jurel (Trachurus murphyi) y caballa (Scomber japonicus). Considerada como una pesquería multiespecífica, la pesquería de cerco puede registrar un alto número de especies en las capturas, más aun, teniendo en cuenta la baja selectividad del arte.
Existe escasa información sobre los factores que influyen sobre la composición de especies en la pesquería de cerco desarrollada sobre pequeños pelágicos, específicamente, de la flota orientada a la captura de anchoveta. Sin embargo, estudios en otras pesquerías describen que la composición de especies en las capturas puede ser influida por i) la selectividad del arte de pesca (Erzini et al. 1999, Woll et al. 2006), ii) el efecto interacción entre dos o más flotas segregadas espacialmente que explotan especies en distintas etapas del ciclo de vida (Coronado et al. 2012), iii) las migraciones nictimerales 
realizadas tanto por especies pelágicas como por los componentes del plancton (Mujica \& Nava 2010) y factores oceanográficos (Csirke et al. 1996, Chávez et al. 2003).

Las principales fluctuaciones ambientales en el área de estudio se asocian a procesos de surgencia costera, y eventos ENOS (Yáñez et al. 2001, Chávez et al. 2003, Parada et al. 2013). Dichos procesos pueden afectar la abundancia y distribución de los recursos pesqueros (Hormazábal et al. 2004, Correa-Ramírez et al. 2007). En este sentido, se ha vinculado una alternancia de especies relacionada con fases decadales frías o cálidas, las que pueden favorecer el crecimiento de anchoveta o sardina española, jurel y caballa, respectivamente (Csirke et al. 1996, Chávez et al. 2003, Alheit \& Ñiquén 2004, Bertrand et al. 2004, Gutiérrez et al. 2007).

Al considerar la importancia económica que posee la anchoveta como recurso pesquero, en conjunto con la dinámica oceanográfica que presenta la zona norte de Chile, es incuestionable que cualquier pieza de información, tendiente a mejorar el conocimiento biológico-pesquero del recurso, es relevante para la comprensión de la dinámica poblacional y el manejo pesquero. Es por esto, que el objetivo del presente estudio fue analizar la composición de especies en los lances que integran la pesquería de cerco de la zona norte de Chile orientada a la captura de anchoveta, y analizar la influencia de factores pesqueros y ecológicos en dicha composición.

\section{Materiales y MÉtodos}

La zona de estudio abarcó desde los $18^{\circ} 23^{\prime} \mathrm{S}$ hasta los $24^{\circ} 00^{\prime} \mathrm{S}$, entre la costa y las 200 millas náuticas. La información utilizada proviene del programa de Seguimiento de Pesquerías Pelágicas de la Zona Norte, desarrollado por el Instituto de Fomento Pesquero (IFOP) entre 2004 y 2012. La actividad fue realizada por flota industrial y artesanal. La flota industrial registra actividad de 60 naves con capacidad de bodega (CB) de $423 \mathrm{~m}^{3}$ y la flota artesanal de 56 naves con CB de $62 \mathrm{~m}^{3}$. En la zona de estudio se utilizan redes de cerco, tipo 'anchovetera', con tamaño de malla en el copo de 5/8 pulgadas. A través del análisis de perfiles de captura, se categorizaron los lances de pesca según la proporción de especies capturadas. Los lances sin captura se eliminaron del análisis.
Como primera etapa, se seleccionaron las especies de mayor importancia según los siguientes criterios, i) representar una captura mayor o igual al 95\% del total de la captura observada en el estrato correspondiente, ii) representar en al menos un lance una captura igual al 100\%, y iii) mediante la aplicación de un HAC (del inglés Hierarchical Agglomerative Clustering, Hartigan 1975), en donde se seleccionaron todas las especies, menos aquellas que se encontraron en la agrupación que se caracterizó por presentar la menor proporción de captura promedio (Deporte et al. 2012). El HAC se aplicó sobre una matriz de proporciones, utilizando la distancia euclidiana y el método de agrupación Ward (Ward 1963). El nivel de corte aplicado sobre el dendrograma, se determinó mediante un scree-test (Cattell 1966) de primer orden (Deporte et al. 2012). Una vez seleccionadas las especies principales, se aplicó un análisis de componentes principales (PCA, siglas en inglés) (Pelletier \& Ferraris 2000), en donde se retuvieron las componentes que explicaron más del 70\% de la varianza (Deporte et al. 2012). Finalmente, sobre las componentes seleccionadas (salida del PCA), se aplicó nuevamente un HAC (Pelletier \& Ferraris 2000), con la misma configuración descrita en la selección de especies. Cada agrupación del dendrograma se consideró como un perfil de captura independiente.

Para analizar la influencia de las distintas variables en la composición de especies, se utilizaron distintos niveles de colapso de la variable respuesta (perfiles de captura). Cuando la variable respuesta consideró 3 y 4 perfiles se aplicó un análisis de regresión multinomial (Friedman et al. 2010). Cuando se consideraron solo 2 perfiles de captura se utilizó un modelo lineal generalizado (GLM) (McCullagh \& Nelder 1989, Nelder \& Wedderburn 1972), con una distribución binomial para la componente aleatoria y una función de enlace del tipo logit. Las variables explicativas fueron: el tipo de flota (artesanal o industrial), la variabilidad temporal (según año, mes y trimestre de recalada), el periodo del día considerando 2 y 4 estados (día-noche y alba-día-crepúsculo-noche), la posición geográfica (latitud y longitud medida en sistema decimal), la temperatura superficial del lance $\left({ }^{\circ} \mathrm{C}\right)$, la zona de pesca y la distancia del lance a la costa expresada como variable continua (distancia en millas náuticas, mn) y variable categórica (DC1: $\leq 1 \mathrm{mn}, \mathrm{DC} 2$ : entre 1 y $5 \mathrm{mn}$, DC3: entre 5 y 10 mn, DC4: > 10 mn).

El procedimiento de análisis consideró un modelo general con todas las variables mencionadas, en el que se realizó una selección de variables mediante la aplicación 
del método Stepwise (Efroymson 1960). El mejor modelo se determinó comparando el valor de Akaike (AIC, Akaike 1974) y el porcentaje de devianza residual explicada por cada modelo. Cabe destacar que, previo al proceso de modelación, la correlación entre dos variables explicativas se estableció cuando el índice de correlación de Pearson fue mayor a 0,5. La selección entre variables correlacionadas para conformar el modelo general se determinó en relación al mayor porcentaje de devianza explicado.

Los análisis se realizaron en la plataforma R Project ( $\mathrm{R}$ Core Team 2014) y las librerías utilizadas fueron amap (Lucas 2014), FactoMineR (Husson et al. 2014), ggplot2 (Wickham 2009), PBSmapping (Schnute et al. 2015), StatDA (Filzmoser 2015), sp (Pebesma et al. 2005), shapefiles (Stabler 2013), mapplots (Gerritsen 2014), psych (Revelle 2017), nnet (Venables \& Ripley 2002) y VGAM (Yee 2017).

\section{Resultados}

La base de datos se constituyó de 9.639 lances de pesca. El número de lances por año se presenta en la Tabla 1. De las 8 especies registradas para el periodo completo (20042012), 5 fueron seleccionadas para el análisis. Estas
Tabla 1. Número de lances utilizados por año y flota. Sin datos para el 2009 / Number of used fishing hauls by year and fleet. No data for 2009

\begin{tabular}{cccccccccc}
\hline & 2004 & 2005 & 2006 & 2007 & 2008 & 2009 & 2010 & 2011 & 2012 \\
\hline Artesanal & 403 & 228 & 134 & 31 & 20 & - & 0 & 3 & 0 \\
Industrial & 1.310 & 1.228 & 679 & 1.206 & 855 & - & 1.073 & 1.381 & 1.088 \\
\hline
\end{tabular}

especies fueron: jurel, caballa, anchoveta, pejerrey (Odontesthes regia) y bacaladillo/mote (Normanichthys crockeri).

Tras aplicar el PCA (Fig. 1), se retuvieron 3 ejes factoriales, los que explicaron el $81,1 \%$ de la varianza. Se establecieron 4 agrupaciones de lances o perfiles de captura (Fig. 2), los que representan un $34,27 \%$ de la varianza. Todas las agrupaciones registraron un porcentaje de captura de anchoveta mayor al 25\%. El perfil 1 (P1) presentó una captura de anchoveta correspondiente al 99,7\%, con escasa presencia de otras especies (Tabla 2). Los perfiles P2 y P3 se consideraron similares, con capturas de anchoveta, jurel y caballa, variando los porcentajes por especie. El P4 se constituyó con lances que presentaron captura de anchoveta, bacaladillo y pejerrey (Fig. 3).
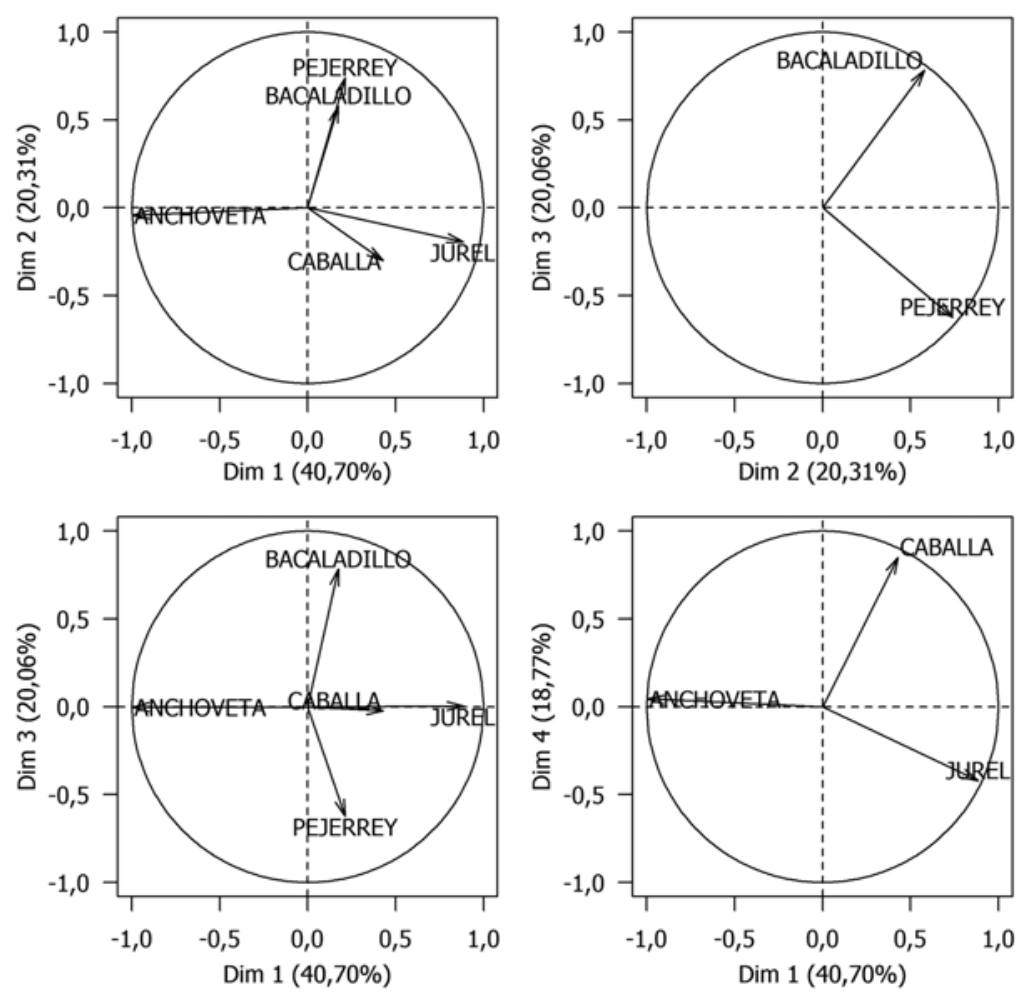

Figura 1. Proyecciones de las especies seleccionadas en los primeros 4 ejes factoriales, cada eje factorial presenta el porcentaje de varianza explicada por el mismo / Projections of the selected species in the 4 first factorials axes, each factorial axes have the percent of variance explained by itself 
Tabla 2. Número de lances y composición porcentual (\%) de especies según agrupación (cluster). En algunas agrupaciones la suma de los porcentajes no es igual al $100 \%(*)$, esto se debe a que el porcentaje ausente corresponde a especies no seleccionadas / Number of fishing hauls and percentual (\%) composition of species by cluster. In some clusters the sum of the percentages is not equal to $100 \%$ $\left({ }^{*}\right)$, that is because the absent percentage corresponds to not selected species

\begin{tabular}{cccccccc}
\hline $\begin{array}{c}N^{\circ} \text { de } \\
\text { cluster }\end{array}$ & $\begin{array}{c}\mathrm{N}^{\circ} \text { lances } \\
\text { por cluster }\end{array}$ & Jurel & Caballa & Anchoveta Pejerrey & Bacaladillo & Total \\
\hline 1 & 9.176 & 0,23 & 0,03 & 99,72 & 0,01 & 0,00 & $99,99^{*}$ \\
2 & 238 & 57,43 & 10,76 & 31,81 & 0,00 & 0,00 & 100,0 \\
3 & 283 & 27,90 & 1,83 & 68,87 & 0,00 & 0,00 & $98,60^{*}$ \\
4 & 73 & 0,23 & 0,26 & 57,25 & 25,92 & 16,33 & 100,0 \\
\hline
\end{tabular}

Las variables que presentaron un alto grado de correlación fueron: mes y trimestre de recalada $(0,97)$, periodo del día con 2 y 4 estados $(0,95)$, latitud y zona de pesca $(-0,92)$, longitud y distancia a la costa, ya sea como variable continua $(-0,96)$ o como variable categórica ($0,50)$. Se esperaba encontrar cierto grado de correlación entre las variables mencionadas, ya que representan la misma información con distinto niveles de agrupación o colapso.

En los modelos que consideraron 4, 3 y 2 perfiles de captura como variable respuesta, al considerar las variables correlacionadas, el mayor porcentaje de devianza fue explicado por el trimestre de recalada, el periodo del día con 4 factores, la zona de pesca y la distancia a la costa establecida como variable categórica (Tabla 3). Dichas variables, en conjunto con el año de recalada, el tipo de flota y la temperatura, conformaron el modelo general (MG). No obstante, justificado por la baja diferencia porcentual de devianza explicada entre el

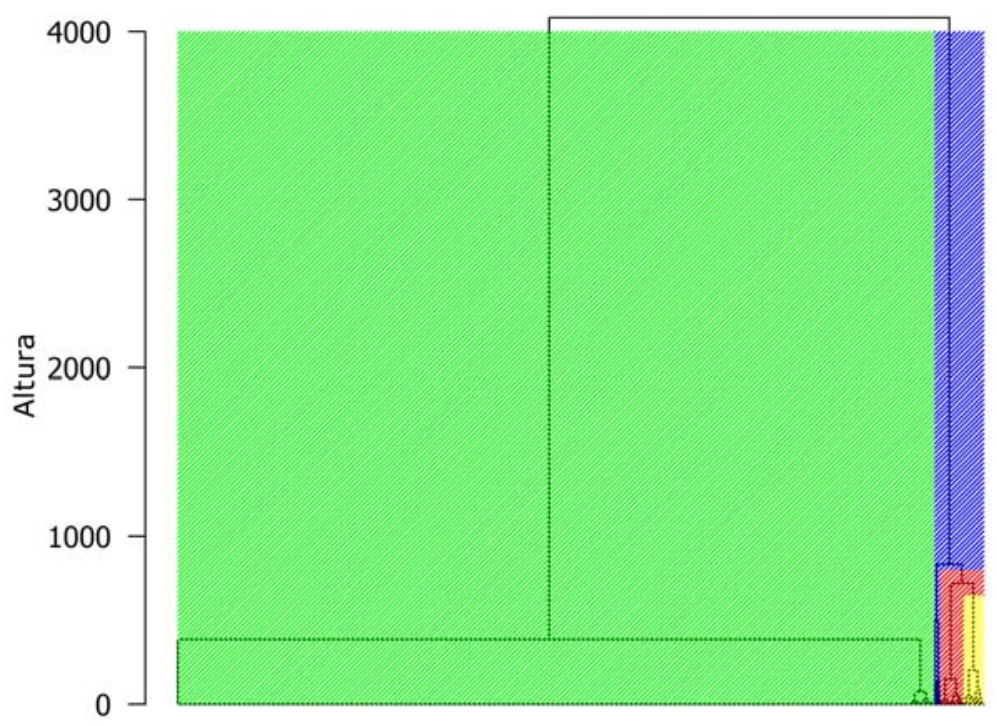

Figura 2. Dendrograma generado por el análisis de aglomeración jerárquico (HAC, siglas en ingles), según la altura se identifican distintos números de agrupaciones con distintos colores, el número de agrupaciones refleja la aproximación utilizada (4 perfiles: verde, azul, rojo y amarillo; 3 perfiles: verde, azul y rojo, y 2 perfiles: verde y azul) / Dendrogram genered by the hierarchichal aglomerative cluster (HAC), according to the height different numbers of clusters with different colors are identified, the cluster number reflects the used approximation (4 profiles: green, blue, red and yellow; 3 profiles: green, blue and red, and 2 profiles: green and blue) 


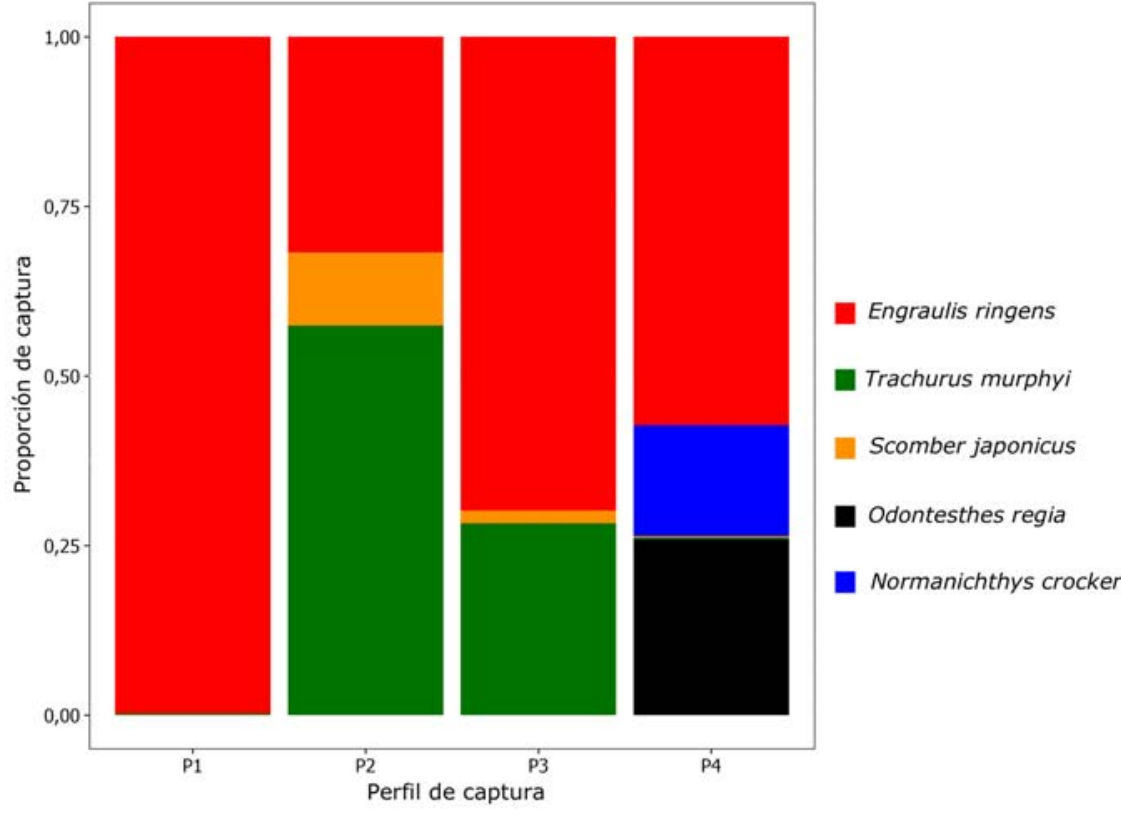

Figura 3. Perfiles de captura identificados en la flota cerquera de la zona norte. Cada color representa una especie / Catch profiles identified in the purse-seine fleet in the northern zone. Each color represent a species

Tabla 3. Porcentaje de devianza residual (\%RD) y valor de Akaike según variable y aproximación utilizada (4, 3 y 2 perfiles de captura). En negro se presentan las variables seleccionadas entre las que presentaron un alto grado de correlación, para conformar el modelo general / Percentage of residual deviance (\%RD) and AIC value by variable and used aproximation ( 4,3 and 2 catch profiles). In black are presented the selected variables among those that presented a high degree of correlation, to conform the general model

\begin{tabular}{|c|c|c|c|c|c|c|c|c|}
\hline & \multicolumn{2}{|c|}{4 perfiles } & \multicolumn{2}{|c|}{3 perfiles } & \multicolumn{2}{|c|}{2 perfiles } & \multicolumn{2}{|c|}{3 perfiles (SS) } \\
\hline & $\%$ RD & $\mathrm{AIC}$ & $\%$ RD & $\mathrm{AIC}$ & $\%$ RD & AIC & $\%$ RD & AIC \\
\hline $\mathrm{MN}^{*}$ & $5.550,9$ & $5.556,9$ & $4.840,6$ & $4.844,6$ & $4.412,0$ & $4.414,0$ & $1.138,9$ & $1.142,9$ \\
\hline $\mathrm{F}$ & 1,26 & $5.492,8$ & 1,42 & $4.779,9$ & 0,03 & $4.414,6$ & 6,03 & $1.078,2$ \\
\hline $\mathrm{AR}$ & 0,13 & $5.555,5$ & 0,06 & $4.845,7$ & 0,00 & $4.415,9$ & 0,93 & $1.136,2$ \\
\hline MR & 0,19 & $5.552,3$ & 0,21 & $4.838,6$ & 0,15 & $4.409,6$ & 0,53 & $1.140,8$ \\
\hline TR & 2,87 & $5.415,8$ & 2,67 & $4.727,1$ & 2,81 & $4.296,0$ & 1,23 & $1.140,9$ \\
\hline PD1 & 0,91 & $5.524,3$ & 0,97 & $4.809,7$ & 0,45 & $4.400,3$ & 2,71 & $1.123,9$ \\
\hline PD2 & 0,81 & $5.518,2$ & 0,87 & $4.806,5$ & 0,40 & $4.398,4$ & 2,38 & $1.119,7$ \\
\hline LAT & 0,88 & $5.514,1$ & 0,98 & $4.801,0$ & 0,34 & $4.401,2$ & 3,89 & $1.102,6$ \\
\hline Z & 1,55 & $5.488,9$ & 1,60 & $4.779,1$ & 1,08 & $4.372,3$ & 3,36 & $1.116,6$ \\
\hline LON & 0,60 & $5.529,7$ & 0,34 & $4.832,0$ & 0,04 & $4.414,3$ & 7,19 & $1.065,0$ \\
\hline D1 & 2,56 & $5.420,9$ & 2,90 & $4.708,2$ & 0,22 & $4.406,3$ & 18,63 & 934,7 \\
\hline D2 & 5,58 & $5.265,0$ & 6,32 & $4.550,6$ & 2,62 & $4.304,2$ & 17,04 & 960,8 \\
\hline $\mathrm{T}$ & 0,50 & $5.535,0$ & 0,55 & $4.821,8$ & 0,40 & $4.398,5$ & 1,15 & $1.133,8$ \\
\hline
\end{tabular}

$\mathrm{MN}^{*}$ : modelo nulo. Se adjunta el valor de AIC y devianza residual total

F: flota, AR: año de recalada, MR: mes de recalada, TR: trimestre de recalada, PD1: periodo del día con 4 estados, PD2: periodo del día con 2 estados, LAT: latitud, Z: zona, LON: longitud, D1: distancia a la costa (variable continua), D2: distancia a la costa (variable categórica), T: temperatura superficial

periodo del día que considera 4 y 2 estados (menor a 0,33\%; Tabla 3), se optó por generar un modelo alterno (MA) con la variable 'periodo del día' de 2 estados. En la Tabla 4 se presenta un resumen de los modelos analizados y del modelo final según la aproximación utilizada.

En la aproximación que considera 4 perfiles de captura, el modelo alterno presentó un menor valor de AIC (Tabla 4), por lo que se seleccionó para continuar con el stepwise. El modelo obtenido (modelo final) conservó todas las variables, con excepción de la variable tipo de flota. Cuando la variable respuesta se agrupó en 3 perfiles de captura, el modelo alterno nuevamente presentó el menor valor de AIC (Tabla 4). Sin embargo, el modelo final mantuvo las variables del modelo alterno. Cuando se consideraron solo 2 perfiles en la variable respuesta, el modelo alterno registró el menor valor de AIC (Tabla 4). El modelo final conservó todas las variables del modelo alterno (Tabla 5). 
Tabla 4. Resumen de los modelos analizados. Por cada modelo se presentan las variables utilizadas, la devianza residual y el valor de AIC / Summary of the models analyzed. For each model are presented the used variables, the residual deviance and the AIC value

\begin{tabular}{|c|c|c|c|c|c|c|c|c|c|c|c|c|c|}
\hline Modelo & NP & $\mathrm{F}$ & $\mathrm{AR}$ & TR & PD1 & PD2 & ZONA & LAT & D1 & D2 & $\mathrm{T}$ & $\begin{array}{c}\text { Devianza } \\
\text { residual }\end{array}$ & AIC \\
\hline MG4 & 4 & & & & & & & & & & & $4.893,9$ & $4.989,9$ \\
\hline MA4 & 4 & & & & & & & & & & & $4.898,6$ & $4.982,6$ \\
\hline MF4 & 4 & & & & & & & & & & & $4.902,1$ & $4.980,1$ \\
\hline MG3 & 3 & & & & & & & & & & & $4.235,8$ & $4.299,8$ \\
\hline MA3 & 3 & & & & & & & & & & & $4.238,6$ & $4.294,6$ \\
\hline MF3 & 3 & & & & & & & & & & & $4.238,6$ & $4.294,6$ \\
\hline MG2 & 2 & & & & & & & & & & & $4.031,7$ & $4.063,7$ \\
\hline MA2 & 2 & & & & & & & & & & & $4.033,0$ & $4.061,0$ \\
\hline MF2 & 2 & & & & & & & & & & & $4.033,0$ & $4.061,0$ \\
\hline MG3* & $3 *$ & & & & & & & & & & & 863,8 & 911,8 \\
\hline MA3* & $3^{*}$ & & & & & & & & & & & 872,6 & 912,6 \\
\hline MF3* & $3 *$ & & & & & & & & & & & 864,6 & 908,6 \\
\hline
\end{tabular}

MG: Modelo general, MA: modelo alterno y MF: modelo final. NP: número de perfiles considerados. *modelos que solo consideran perfiles P2, P3 y P4

En relación a la capacidad predictiva de los modelos finales, en la aproximación que consideró 4 y 3 perfiles de captura, se estimó en todos los lances una alta probabilidad de pertenecer a P1 (lances con captura $\sim 100 \%$ de anchoveta, Tabla 2). Ambas aproximaciones presentaron una tasa de error de predicción del 6,07\%, correspondiente al número de lances categorizados originalmente como cualquier perfil distinto de P1. Cuando se consideraron sólo 2 perfiles de captura, también se categorizaron todos los lances como $\mathrm{P} 1$, ya que el valor más alto en la probabilidad estimada de que el lance sea distinto que P1 fue de 0,37.

Ningún modelo fue capaz de predecir correctamente el perfil de captura asociado a los lances de P2, P3 y P4, por lo que se evaluó una cuarta aproximación, en donde se modeló una submuestra constituida solo con los lances de P2, P3 y P4 ('3 Perfiles (SS)' en Tabla 3 y MG3*, MA3*, y MF3* en Tabla 4).

En relación a las variables seleccionadas para conformar el modelo general, la cuarta aproximación presentó un mayor porcentaje de devianza explicada por las variables LAT y D1 en comparación a lo observado en las otras aproximaciones (Tabla 3). Para mantener la estructura de análisis, se continuó evaluando un modelo general y otro alterno (considerando PD1 y PD2 respectivamente). En esta oportunidad, el modelo general presentó el menor valor de AIC (Tabla 4) y según el stepwise, se eliminó del modelo la variable Flota.
Tabla 5. Parámetros estimados por el modelo binomial MF2 (Tabla 4). Abreviaciones explicadas en Tabla 3. Para las variables TR, PD2, Z y D2 el guion indica la relación de la categoría analizada en relación a la categoría de referencia / Estimated parameters for the MF2 binomial model (Table 4). Abbrevations are explained in Table 3. For TR, PD2, Z and D2 variables the script indicates the relationship between the analized category and the reference category

\begin{tabular}{|c|c|c|c|c|c|c|}
\hline Variable & Estimado & $\begin{array}{c}\text { Error } \\
\text { estándar }\end{array}$ & $\mathrm{Z}$ & $\operatorname{Pr}(>|z|)$ & & \\
\hline (Intercepto) & 57,14521 & 33,18001 & 1,722 & 0,085019 & . & \\
\hline $\mathrm{F}$ & $-0,41537$ & 0,22670 & $-1,832$ & 0,066919 & . & \\
\hline $\mathrm{AR}$ & $-0,03134$ & 0,01661 & $-1,887$ & 0,059172 & . & \\
\hline TR - 2 & 1,24801 & 0,13643 & 9,148 & $<2 \mathrm{e}-16$ & *** & \\
\hline TR - 3 & 0,57088 & 0,17243 & 3,311 & 0,000930 & $* * *$ & \\
\hline TR - 4 & $-0,29449$ & 0,15203 & $-1,937$ & 0,052735 & . & \\
\hline PD2 - N & $-0,32048$ & 0,09469 & $-3,385$ & 0,000713 & $* * *$ & \\
\hline$Z-2$ & 0,94865 & 0,11442 & 8,291 & $<2 \mathrm{e}-16$ & $* * *$ & \\
\hline$Z-3$ & 0,76402 & 0,14321 & 5,335 & $9,55 \mathrm{e}-08$ & $* * *$ & \\
\hline$Z-4$ & 0,56910 & 1,04668 & 0,544 & 0,586634 & & \\
\hline D2 - 2 & $-1,60792$ & 0,35612 & $-4,515$ & $6,33 \mathrm{e}-06$ & *** & \\
\hline D2 - 3 & $-1,50052$ & 0,37198 & $-4,034$ & $5,49 \mathrm{e}-05$ & $* * *$ & \\
\hline D2 - 4 & $-0,57134$ & 0,36749 & $-1,555$ & 0,120014 & & \\
\hline $\mathrm{T}$ & 0,18764 & 0,03234 & 5,802 & $6,54 \mathrm{e}-09$ & $* * *$ & \\
\hline Código de significancia & $0^{\prime * * * *^{\prime}}$ & $0,001^{' * * \prime}$ & $0,01 * '$ & $0,05^{\prime}$ & $0,1^{\cdots}$ & 1 \\
\hline Devianza nula & 4412 & & & & & \\
\hline Devianza residual & 4033 & & & & & \\
\hline AIC & 4061 & & & & & \\
\hline
\end{tabular}


En la Tabla 6 se presentan los perfiles predichos por el modelo final (MF3*) en comparación a los perfiles establecidos ('observados’). Se observó una tasa de error de predicción del 37,26\%.

\section{Discusión}

En la pesquería de cerco de la zona norte de Chile, se identificaron 4 grupos de lances o perfiles de captura, de los cuales, el primer grupo fue el de mayor tamaño ( $100 \%$ captura de anchoveta), agrupando el 94\% de los lances analizados. Solo el 6\% de los lances se categorizó como un perfil de captura mixta (distinto de P1). En este sentido, si bien se considera que el arte de cerco es poco selectivo, existen múltiples factores que pueden influir sobre la proporción de especies. Dentro de estos, es posible señalar la profundidad de calado del lance, distribución vertical del cardumen, la zona de pesca, la hora del lance, cambios en la distribución horizontal y vertical de las especies (Lokkeborg \& Bjorda 1992, Lanz et al. 2008, Raeisi et al. 2011), la interacción de las flotas artesanal e industrial con ámbitos de acción espacial diferente (Coronado et al. 2012) y factores biológicos como reproducción y alimentación (Cushing 1977, Fréon et al. 1992). Sin embargo, uno de los principales factores que probablemente se relacionan con la baja proporción de fauna acompañante en la pesquería en análisis, podría ser la biología de la anchoveta y su comportamiento gregario tendiente a formar cardúmenes compactos de

Tabla 6. Categorización de lances (predicciones) según el modelo MF3* en comparación con la categorización establecida ('observada') de los lances respectivos. El modelo sólo considera los perfiles P2, P3 y P4 / Categorization of the fishing hauls (predictions) according to MF3* model in comparison to the established categorization ('observed') of the respective fishing hauls. The models just consider the P2, P3 and P4 profiles

\begin{tabular}{lccc}
\hline & $\begin{array}{c}\text { P2 } \\
\text { estimado }\end{array}$ & $\begin{array}{c}\text { P3 } \\
\text { estimado }\end{array}$ & $\begin{array}{c}\text { P4 } \\
\text { estimado }\end{array}$ \\
\hline P2 observado & 135 & 89 & 12 \\
P3 observado & 88 & 176 & 15 \\
P4 observado & 2 & 12 & 56 \\
\hline
\end{tabular}

300 peces por $\mathrm{m}^{3}$ (Simmonds et al. 1992), que junto a la experiencia de los pescadores en la interpretación de los ecogramas (Rose \& Leggett 1988, Scalabrin 1997), permitiría la ocurrencia de una captura con baja proporción de fauna acompañante (Fréon \& Misund 1999).

En los resultados del modelamiento con dos perfiles, lances puros (con captura $\sim 100 \%$ de anchoveta) y lances mixtos (con captura de fauna acompañante), las variables de mayor significancia en el modelo (MG2, Tabla 4) fueron el trimestre de recalada, el periodo diurno, la zona de pesca, la distancia a la costa y la temperatura. La influencia del periodo del día, podría vincularse con el patrón de migración vertical de los pequeños pelágicos, los que se dispersan durante la noche, generando grupos de baja densidad cercanos a la superficie, y que al amanecer se reagrupan manteniendo este comportamiento a lo largo del día (Azzali et al. 1985, Fréon et al. 1996, Parrish \& Hamner 1997, Fréon \& Misund 1999, Cardinale et al. 2003, Kaltenberg \& Benoit-Bird 2009). Si bien las fuerzas que gatillan la formación y dinámica del cardumen no se entienden bien, se cree que estaría regulado por factores como alimentación y depredación. Según Pitcher \& Parrish (1993), el comportamiento de formación de cardumen aumenta la probabilidad de encuentro con la presa, sin embargo, esta disminuye cuando la presa tiene la capacidad de migrar fuera del alcance del predador, por lo que la formación de cardúmenes de anchoveta por la noche podría presentar una ventaja en la alimentación cuando el hábitat de la anchoveta se superpone con su presa (Kaltenberg \& Benoit-Bird 2009). La anchoveta es considerada un depredador generalista y oportunista dependiendo de la disponibilidad de presas en el ambiente (Bertrand et al. 2008¹, Espinoza \& Bertrand 2008, Medina et al. 2015), comportamiento que podría influir en la formación del cardumen, para aumentar la probabilidad de alimentación y disminuir el grado de vulnerabilidad ante los predadores.

\footnotetext{
${ }^{1}$ Bertrand A, A Chaigneau, JC Coetzee, M Gutiérrez, J Habasque, L Hutchings, J Ledesma, S Peraltilla \& CD Van der Lingen. 2008. Does the vertical extent of suitable physical habitat constrain small pelagic fish populations in the Humboldt and Benguela Current upwelling systems International Symposium on Eastern boundary upwelling ecosystems: integrative and comparative approaches. Las Palmas, Canary Islands, Spain, 2-6 June 2008. Abstracts Book, pp. 201-202.
} 
La temperatura es otra variable de importancia, al reflejar la variabilidad estacional e inter anual (ENOS), afectando la distribución de especies. La anchoveta entre el periodo primavera-verano se desplaza a zonas costeras (Yáñez et al. 2001, Bertrand et al. 2004) asociadas a un mayor gradiente de temperatura, ampliando su rango de distribución latitudinal hacia el sur (Yáñez et al. 1995, Ñiquen \& Bouchon 2004). No obstante, durante el periodo otoño-invierno, se dispersa y desplaza hacia zonas oceánicas. Dicho comportamiento puede ser intensificado o debilitado por la ocurrencia de los eventos El Niño o La Niña (Parada et al. 2013).

El trimestre de recalada, la zona, y la distancia a la costa son variables que explican la composición de los perfiles de captura, ya que caracterizan espacial y temporalmente al perfil de captura. En términos de evaluar los factores del modelo, el cuarto trimestre presentó una influencia levemente significativa sobre la devianza explicada en relación a lo explicado por el primer trimestre. No se observó una influencia significativa en la devianza explicada por parte de la zona 4 en relación a lo explicado por la zona 1, probablemente debido al bajo número de lances registrados en dicha zona (0,28\% de los lances). La importancia de estas variables podría estar relacionada con la dinámica espacial y temporal de la flota que opera en zonas costeras de los puertos de Arica e Iquique entre enero y marzo, desplazándose al sur de Iquique entre abril y julio, en tanto durante el último trimestre del año, la flota opera más alejada de la costa. Interanualmente durante el invierno del 2007 por el efecto de La Niña, la flota operó a mayores distancias de la costa debido al desplazamiento de la anchoveta hacia áreas más oceánicas, en tanto en el 2006, la zona fue afectada por un calentamiento del agua con un accionar más costero de la flota, registrándose especies como el jurel y la caballa, asociadas a temperaturas más cálidas (Chávez et al. 2003).

En conclusión, los grupos de captura responden en parte a los periodos del día, por el comportamiento gregario de la anchoveta, sin embargo, la variabilidad ambiental en la zona parece ser un factor determinante en la influencia registrada por el año y la distancia a la costa.

\section{Agradecimientos}

Los autores agradecen a los observadores científicos (OC) del Instituto de Fomento Pesquero (IFOP) por el monitoreo de las capturas realizado a bordo de naves pesqueras en la zona Norte de Chile. Los autores también agradecen los comentarios y aportes brindados por Mauricio Ibarra.

\section{LITERATURA CITADA}

Akaike H. 1974. A new look at the statistical model identification. IEEE Transactions on Automatic Control 19(6): 716-723.

Alheit $\mathbf{J}$ \& $\mathbf{M}$ Ñiquen. 2004. Regime shift in the Humboldt Current ecosystem. Progress in Oceanography 60: 201222.

Azzali M, G Buracchi, S Conti, S Gambetti \& M Luna. 1985. Relationship between the forms of pelagic fish distribution and nictemeral periods. A tentative model of behaviour. Oebalia 11: 471-488.

Bertrand A, M Segura, M Gutiérrez \& L Vásquez. 2004. From small-scale habitat loopholes to decadal cycles: a habitat-based hypothesis explaining fluctuation in pelagic fish populations off Peru. Fish and Fisheries 5: 296-316.

Cardinale M, M Casini, F Arrhenius \& N Hakkansson. 2003. Diel spatial distribution and feeding activity of herring Clupea harengus and sprat Sprattus sprattus in the Baltic Sea. Aquatic Living Resources 16: 283-292.

Cattell R. 1966. The scree test for the number of factors. Multivariate Behavioral Research 1: 245-276.

Chávez F, J Ryan, S Lluch-Cota \& M Ñiquen. 2003. From anchovies to sardines and back: Multidecadal change in the Pacific Ocean. Science 299(5604): 217-221.

Coronado E, S Castro \& T Brulé. 2012. Estimación de la composición de las capturas y la cuasi-renta de diferentes flotas dirigidas a la pesquería de Mero Rojo en Yucatán, México. Proceedings of the 65th Gulf and Caribbean Fisheries Institute GCFI 65: 373-383.

Correa-Ramirez MA, S Hormazabal \& G Yuras. 2007. Mesoscale eddies and high chlorophyll concentrations off central Chile $\left(29^{\circ}-39^{\circ} \mathrm{S}\right)$. Geophysical Research Letters 34(12), L12604.<https://doi.org/10.1029/2007GL029541>.

Csirke J, R Guevara-Carrasco, G Cárdenas, M Ñiquen \& A Chipollini. 1996. Situación de los recursos anchoveta (Engraulis ringens) y sardina (Sardinops sagax) a principios de 1994 y perspectivas para la pesca en el Perú, con particular referencia a las regiones norte y centro de la costa peruana. Boletín del Instituto del Mar del Perú 15(1): $1-23$.

Cushing D. 1977. Observations on ûsh shoals with the ARL scanner. Rapports et Procès-Verbaux des Réunions, Conseil de International Exploration de la Mer 170: 15-20. 
Deporte N, C Ulrich, S Mahévas, S Demanèche \& F Bastardie. 2012. Regional métier definition: a comparative investigation of statistical methods using a workflow applied to international otter trawl fisheries in the North Sea. ICES Journal of Marine Science 692: 331-342.

Efroymson MA. 1960. Multiple regression analysis In: Ralston A \& HS Wilf (eds). Mathematical methods for digital computers, pp. 191-203. John Wiley \& Sons, New York.

Espinoza P \& A Bertrand. 2008. Revisiting Peruvian anchovy (Engraulis ringens) trophodynamics provides a new vision of the Humboldt Current System. Progress in Oceanography 79: 215-227.

Erzini K, J Goncalves, L Bentes, P Lino \& J Ribeiro. 1999. Catch composition, catch rates and size selectivity of three long-line methods in the Algarve (southern Portugal). Boletín, Instituto Español de Oceanografía 15: 313-323.

Filzmoser P. 2015. StatDA: Statistical Analysis for Environmental Data. R package version 1.6.9. <http://cran.rproject.org/web/packages/StatDA/StatDA.pdf $>$

Fréon P \& O Misund. 1999. Dynamics of pelagic fish distribution and behaviour: Effect on fisheries and stock assessment, 348 pp. Fishing New Books, Blackwell Science Ltd, Oxford.

Fréon P, F Gerlotto \& M Soria. 1992. Changes in school structure according to external stimuli: Description and influence on acoustic assessment. Fisheries Research 15: 45-66.

Fréon P, F Gerlotto \& M Soria. 1996. Diel variability of school structure with special reference to transition periods. ICES Journal of Marine Science 53: 459-464.

Friedman J, T Hastie \& R Tibshirani. 2010. Regularization paths for generalized linear models via coordinate descent. Journal of Statistical Software 33(1): 1-22.

Gerritsen H. 2014. mapplots: Data visualisation on maps. R package version 1.5. <http://CRAN.R-project.org/ package $=$ mapplots $>$

Gutiérrez M, G Swartzman, A Bertrand \& S Bertrand. 2007. Anchovy and sardine spatial dynamics and aggregation patterns in the Humboldt Current ecosystem, Peru, from 1983 to 2003. Fisheries Oceanography 16: 155168.

Hartigan JA. 1975. Clustering algorithms, 366 pp. John Wiley, New York.

Hormazábal S, G Shaffer \& O Leth. 2004. The coastal transition zone off Chile. Journal of Geophysical Research 109, C01021. <doi:10.1029 2003JC001956>

Husson F, J Josse, S Le \& J Mazet. 2014. FactoMineR: Multivariate exploratory data analysis and data mining with R. R package version 1.26.<http://factominer.free.fr>

Kaltenberg A \& K Benoit-Bird. 2009. Diel behavior of sardine and anchovy schools in the California Current System. Marine Ecology Progress Series 394: 247-262. <doi: 10.3354/meps08252>
Lanz E, M Nevárez-Martinez, J López-Martinez \& J Dworar. 2008. Spatial distribution and species composition of small pelagic fishes in the Gulf of California. Revista de Biología Tropical 56(2): 575-590.

Løkkeborg S \& A Bjorda. 1992. Species and size selectivity in long-line fishing: a review. Fisheries Research 13: 311322.

Lucas A. 2014. amap: Another Multidimensional Analysis Package. Versión 0.8-14. <https://CRAN.R-project.org/ package $=$ amap $>$

McCullagh P \& JA Nelder. 1989. Generalized linear models. Monographs on statistics and applied probabibilty, 511 pp. Chapman and Hall, London.

Medina M, L Herrera, J Castillo, J Jaque \& N Pizarro. 2015. Alimentación de la anchoveta (Engraulis ringens) en el norte de Chile $\left(18^{\circ} 25^{\prime}-25^{\circ} 40^{\prime} \mathrm{S}\right.$ ) en diciembre de 2010 . Latin American Journal of Aquatic Research 43(1): 46-58.

Mujica A \& M Nava. 2010. Distribución espacial de larvas de crustáceos decápodos planctónicos en canales orientales de la isla Chiloé, Chile. Latin American Journal of Aquatic Research 38(1): 95-106.

Nelder J \& R Wedderburn. 1972. Generalized linear models. Journal of the Royal Statistical Society A 135: 370-384.

Ñiquen M \& M Bouchon. 2004. Impact of El Niño event on pelagic fisheries in Peruvian waters. Deep-Sea Research II 51: 563-574.

Parada C, B Yannicelli, S Hormazábal, S Vásquez, J Porobiæ, B Ernst, C Gatica, M Arteaga, A Montecinos, S Núñez \& A Gretchina. 2013. Variabilidad ambiental y recursos pesqueros en el Pacífico suroriental: estado de la investigación y desafíos para el manejo pesquero. Latin American Journal of Aquatic Research 41(1): 1-28.

Parrish JK \& WM Hammer. 1997. Animal groups in three dimensions, 378 pp. Cambridge University Press, Cambridge.

Pelletier D \& J Ferraris. 2000. A multivariate approach for defining fishing tactics from commercial catch and effort data. Canadian Journal of Fisheries and Aquatic Science 57: 51-65.

Pitcher T \& J Parrish. 1993. The functions of shoaling behaviour. In: Pitcher TJ (ed). The behaviour of teleost fishes, pp. 363-439. Chapman and Hall, London.

R Core Team. 2014. R: A language and environment for statistical computing. R Foundation for Statistical Computing, Vienna. <http://www.R-project.org/>

Raeisi H, S Hosseini, S Paighambari, S Taghavi \& $\mathbf{R}$ Davoodi. 2011. Species composition and depth variation of cutlassfish Trichiurus lepturus L. 1785) trawl bycatch in the fishing grounds of Bushehr waters, Persian Gulf. African Journal of Biotechnology 10(76): 17610-17619.

Revelle W. 2017. Psych: Procedures for Personality and Psychological Research. Version 1.7.8. Northwestern University, Evanston. <https://CRAN.R-project.org/ package $=$ psych $>$. 
Rose GA \& WC Leggett. 1988. Hydroacoustic signal classication of sh schools by species. Canadian Journal of Fisheries and Aquatic Sciences 45: 597-604.

Scalabrin C. 1997. Identication acoustique des espèces pélagiques à partir d'attributs discriminants des bancs de poissons monospéciques. Thèse $\mathrm{PhD}$. Océanographie Biologique, Université de Bretagne Occidentale, Brest, 244 pp.

Schnute J, N Boers, R Haigh \& A Couture-Beil. 2015. PBSmapping 2.69: User's guide. Canadian Technical Report of Fisheries and Aquatic Sciences 2549: 1-43.

Simmonds E, NJ Williamson, F Gerlotto \& A Aglen. 1992. Acoustic survey design and analysis procedures: a comprehensive review of current practice. ICES Cooperative Research Reports 187: 1-127.

Stabler B. 2013. shapefiles: Read and Write ESRI Shapefiles $<$ https://cran.r-project.org/web/packages/shapefiles/ index.html>
Venables W \& B Ripley. 2002. Modern Applied Statistics with S, 498 pp. Springer, New York.

Ward J. 1963. Hierarchical grouping to optimize an objective function. Journal of the American Statistical Association 58: 236-244.

Woll A, G Van der Meeren \& I Fossen. 2006. Spatial variation in abundance and catch composition of Cancer pagurus in Norwegian waters: biological reasoning and implications for assessment. ICES Journal of Marine Science 63: 421433.

Yáñez E, A González \& M Barbieri. 1995. Estructura térmica superficial del mar asociada a la distribución espaciotemporal de sardina y anchoveta en la zona norte de Chile entre 1987 y 1992. Investigaciones Marinas 23: 123-147.

Yáñez E, MA Barbieri, C Silva, K Nieto \& F Espíndola. 2001. Climate variability and pelagic fisheries in northern Chile. Progress in Oceanography 49: 581-596.

Yee TW. 2017. VGAM: Vector Generalized Linear and Additive Models. R package version 1.0-4, <https://CRAN.Rproject.org/package $=$ VGAM $>$

Recibido el 3 de octubre de 2016 y aceptado el 20 de marzo de 2018

Editor Asociado: Mauricio Landaeta D. 